\title{
A Rectangular Waveguide-to-Coplanar Waveguide Transition at D-band Using Wideband Patch Antenna
}

\author{
Dong, Yunfeng; Johansen, Tom Keinicke; Zhurbenko, Vitaliy; Hanberg, Peter Jesper
}

Published in:

Proceedings of the 48th European Microwave Conference

Link to article, DOI:

10.23919/EuMC.2018.8541505

Publication date:

2018

Document Version

Peer reviewed version

Link back to DTU Orbit

Citation (APA):

Dong, Y., Johansen, T. K., Zhurbenko, V., \& Hanberg, P. J. (2018). A Rectangular Waveguide-to-Coplanar Waveguide Transition at D-band Using Wideband Patch Antenna. In Proceedings of the 48th European Microwave Conference (pp. 1045-1048). IEEE. https://doi.org/10.23919/EuMC.2018.8541505

\section{General rights}

Copyright and moral rights for the publications made accessible in the public portal are retained by the authors and/or other copyright owners and it is a condition of accessing publications that users recognise and abide by the legal requirements associated with these rights.

- Users may download and print one copy of any publication from the public portal for the purpose of private study or research.

- You may not further distribute the material or use it for any profit-making activity or commercial gain

- You may freely distribute the URL identifying the publication in the public portal 


\title{
A Rectangular Waveguide-to-Coplanar Waveguide Transition at D-band Using Wideband Patch Antenna
}

\author{
Yunfeng Dong ${ }^{\# 1}$, Tom K. Johansen ${ }^{\# 2}$, Vitaliy Zhurbenko ${ }^{\# 3}$, and Peter Jesper Hanberg*4 \\ \#Electromagnetic Systems, Technical University of Denmark, Kgs. Lyngby, Denmark \\ ${ }^{*}$ DTU Danchip, Technical University of Denmark, Kgs. Lyngby, Denmark \\ $\left\{{ }^{1}\right.$ yundon, ${ }^{2}$ tkj, $\left.{ }^{3} \mathrm{vz}\right\} @$ elektro.dtu.dk, ${ }^{4}$ jehan@danchip.dtu.dk
}

\begin{abstract}
This paper presents the design of a transition at D-band (110-170 GHz) between rectangular waveguide and coplanar waveguide (CPW) using wideband patch antenna. With the rectangular ring structure, the proposed patch antenna is specialized for high gain and large bandwidth which can be used for wireless chip-to-chip communication or implemented as a rectangular waveguide-to-CPW transition. A simulated gain of $7.4 \mathrm{dBi}$ with $36 \%$ bandwidth centered at $140 \mathrm{GHz}$ is achieved. The fabricated rectangular waveguide-to-CPW transition in a back-to-back configuration exhibits a bandwidth of $42.2 \mathrm{GHz}$ at D-band. From $118.8 \mathrm{GHz}$ to $161 \mathrm{GHz}$, the return loss is better than $10 \mathrm{~dB}$ and each fabricated rectangular waveguide-to-CPW transition introduces less than $2 \mathrm{~dB}$ insertion loss.
\end{abstract}

Keywords-patch antennas, ultra wideband antennas, wireless communication, integrated circuit packaging.

\section{INTRODUCTION}

With the ever increasing demand worldwide for high-speed data transmission, wideband antennas have been widely used for wireless chip-to-chip communication as well as system integration and packaging at millimeter-wave and submillimeterwave frequencies. However, not all types of antennas are suitable since the chips at such high frequencies mainly rely on planar structures due to their fabrication processes. The designs and implementations of wideband antennas are normally subjected to several concerns including the size, substrate, radiation characteristics, and operating bandwidth. The challenge is to achieve a high gain and at the same time a large bandwidth within a limited occupation area. Besides, the designed antenna should be compatible with the planar transmission lines used for the circuits, otherwise coplanar transitions might have to be added which introduce extra discontinuities and losses. As is reported in [1], a bandwidth of $29 \mathrm{GHz}$ is achieved by the proposed Yagi antenna at Wband $(75-110 \mathrm{GHz})$ which corresponds to $31.4 \%$ bandwidth while the gain is around $0.4 \mathrm{dBi}$ at $94 \mathrm{GHz}$. In [2], by placing the stacked dielectric resonators on the top of the proposed meander slot antenna, a measured gain of $4.7 \mathrm{dBi}$ is achieved at $130 \mathrm{GHz}$ with $11 \%$ bandwidth. The directivity of the wideband antenna can be further increased by adding a hemisphere silicon lens on the top of the substrate or using an array with feeding networks while either extra chip-level assembly or large occupation area is required [3]-[5].

Fig. 1 shows the envisaged structure of the proposed wideband patch antenna when it is integrated on a chip. Conventional patch antenna has a certain dimension related to the operating frequency and simple planar structure which can be easily connected to different planar transmission lines. Being improved, the proposed patch antenna shown in Fig. 1 has a

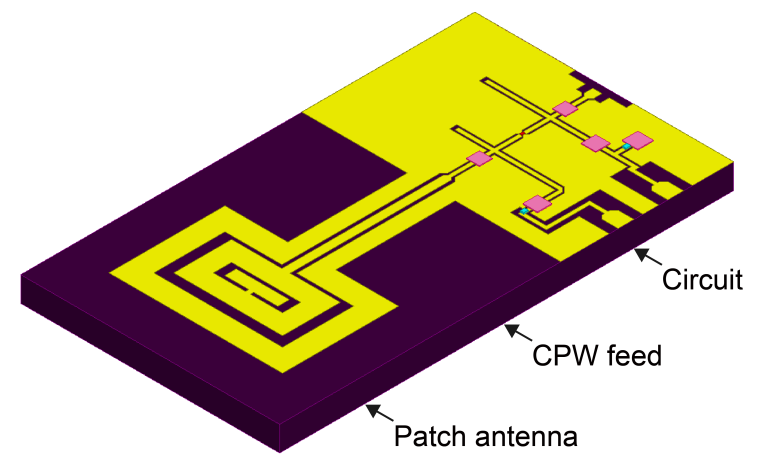

Fig. 1. Envisaged structure of the wideband patch antenna when it is integrated on a chip (circuit from [6]).

rectangular ring structure which increases the gain and enlarges the bandwidth without adding extra component. Besides, it is fed by CPW directly where the rectangular patch at the center is connected to the signal trace and the ground traces are designed as a part of the patch antenna. Apart from being used for wireless chip-to-chip communication, the proposed wideband patch antenna can also be implemented as a rectangular waveguide-to-CPW transition for system integration and packaging. The waveguide package not only provides isolation and protection to the chip but also serves as the standardized interface for connecting with other components and systems.

In this work, a wideband patch antenna for D-band applications is designed and analyzed by using High Frequency Structural Simulator (HFSS). In order to validate the concept, an antenna prototype based on quartz substrate is implemented which can be reconfigured for other substrates such as gallium arsenide (GaAs) or indium phosphide (InP). In Section II, the design of wideband patch antenna for wireless chip-tochip communication is described. Besides, the simulated return loss and the far-field radiation characteristics are explained. In Section III, the optimization of the wideband patch antenna and the design of rectangular waveguide-to-CPW transition in a back-to-back configuration are demonstrated. In Section $\mathrm{IV}$, the fabricated rectangular waveguide-to-CPW transition in a back-to-back configuration is shown and the measurement results are compared with the simulation results.

\section{Wideband PATCH ANTENNA FOR Wireless CHIP-TO-CHIP COMMUNICATION}

The wideband patch antenna shown in Fig. 2 is designed for wireless chip-to-chip communication. In the communication link, the transceiver chips are aligned towards each other in the 

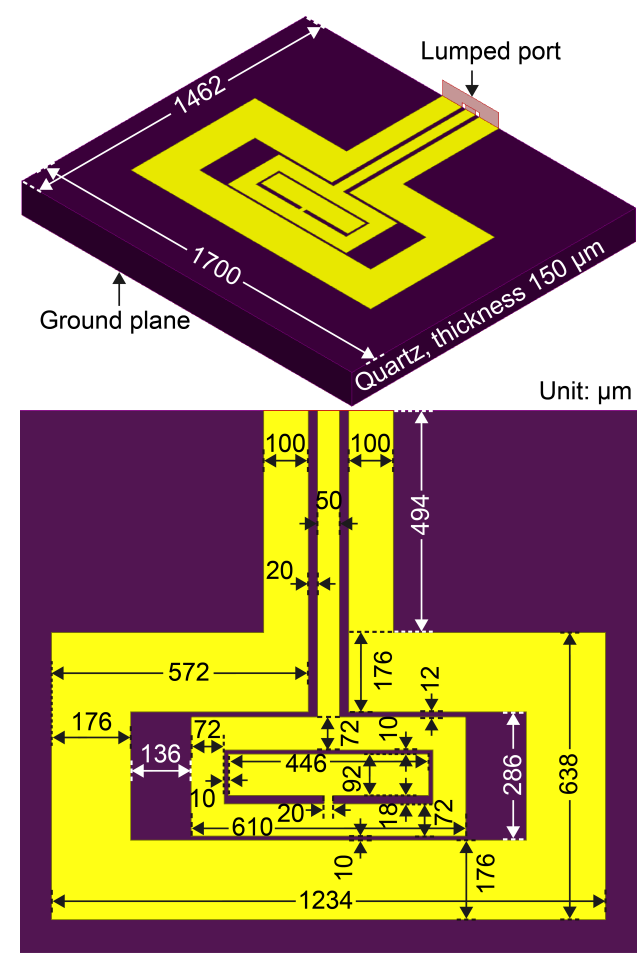

Fig. 2. Designed wideband patch antenna for wireless chip-to-chip communication.

direction of maximum radiation and the packaging structure can be avoided. The designed wideband patch antenna is patterned on a conductor layer which is located on the top of the quartz substrate. Besides, another conductor layer is added on the bottom which serves as a ground plane. The quartz substrate has a thickness of $150 \mu \mathrm{m}$ and the material of the conductor layer is gold. Due to the skin effect, the thickness of the conductor layer should be more than the skin depth at the lower frequency limit of D-band. As a result, the conductor layer with a thickness of $400 \mathrm{~nm}$ is used for designing the wideband patch antenna.

As a part of the wideband patch antenna, a $50 \Omega \mathrm{CPW}$ feed with a length of $670 \mu \mathrm{m}$ is included in the design which makes it possible for being integrated on-chip or connected with other circuits based on CPW. The width of the signal trace is $50 \mu \mathrm{m}$ and the width of the ground traces is $100 \mu \mathrm{m}$ with a gap width of $20 \mu \mathrm{m}$. The signal trace is connected to a patch which is cut into a rectangular ring connecting to a smaller rectangular patch at the center of the patch geometry. The ground traces are tapered out from $100 \mu \mathrm{m}$ to $176 \mu \mathrm{m}$ around the patch geometry forming another rectangular ring. The dimensions of the wideband patch antenna as well as the quartz substrate are summarized in Fig. 2. They are optimized for achieving the maximum bandwidth and gain at D-band. In order to simulate the far-field radiation characteristics, the coordinate system is assigned to the center of the patch geometry and an air box is added around the designed wideband patch antenna which is large enough reaching the antenna far-field region. Lumped port with vertical perfect electric conductor (PEC) bridge is used as the excitation scheme where the PEC bridge touches the ground traces and the port is assigned to a sheet between the signal trace and the PEC bridge. The parasitic inductance of the port is calibrated out from the simulation results.

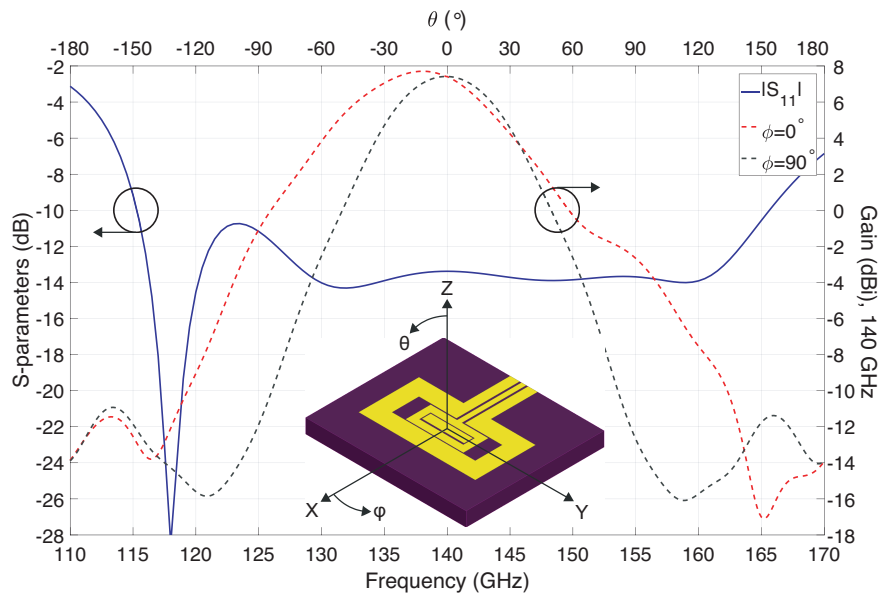

Fig. 3. Simulated return loss at D-band and far-field radiation patterns of the wideband patch antenna at $140 \mathrm{GHz}$.

The solid line in Fig. 3 shows the simulated return loss of the wideband patch antenna at D-band. With the rectangular ring structure, the wideband patch antenna achieves a bandwidth of $50.5 \mathrm{GHz}$ ranging from $115.3 \mathrm{GHz}$ to $165.6 \mathrm{GHz}$ in which the simulated return loss remains better than $10 \mathrm{~dB}$ and it corresponds to $36 \%$ bandwidth centered at $140 \mathrm{GHz}$. The dashed lines in Fig. 3 show the simulated far-field radiation patterns of the wideband patch patch antenna at $140 \mathrm{GHz}$ when $\phi$ equals $0^{\circ}$ and $90^{\circ}$, respectively. A simulated antenna gain of $7.4 \mathrm{dBi}$ is achieved in the direction perpendicular to the wideband patch antenna where $\theta$ and $\phi$ are $0^{\circ}$. Compared with the conventional patch antenna at D-band, larger bandwidth and higher gain can be provided by the proposed patch antenna while the occupation area is comparable.

\section{Wideband PATCH ANTENNA FOR ReCtANGUlaR WAVEGUIDE-TO-CPW TRANSITION}

For system integration and packaging, the proposed wideband patch antenna can be implemented as a rectangular waveguide-to-CPW transition. Since rectangular waveguide serves as a standardized interface for connecting with other components and systems, the gain of the system can be further increased. As is reported in [7], the wireless chip-to-chip communication is realized by connecting a horn antenna to a rectangular waveguide-to-CPW transition which provides higher gain and larger bandwidth in comparison to the patch antenna itself. Fig. 4 shows the packaging structure of the proposed rectangular waveguide-to-CPW transition at D-band using wideband patch antenna.

The packaging structure shown in Fig. 4 is in a back-toback configuration where WR-6.5 rectangular waveguides are used as the input and output guiding electromagnetic waves. At the beginning, the rectangular waveguide turns $90^{\circ}$ towards the transition in the direction perpendicular to the wideband patch antenna. Besides, in order to guide electromagnetic waves smoothly and overcome the discontinuities, a tapered waveguide structure with a length of $1000 \mu \mathrm{m}$ is introduced where the width is kept constant and the height is reduced from $825.5 \mu \mathrm{m}$ to $680 \mu \mathrm{m}$. The wideband patch antenna is located at the end of the rectangular waveguide. The depth of the cavity is $150 \mu \mathrm{m}$ which equals the thickness of the quartz substrate. 


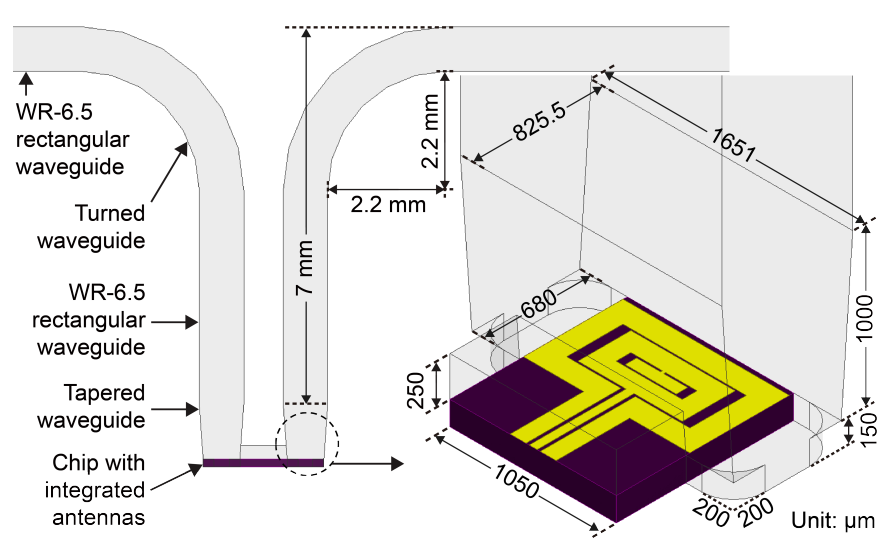

Fig. 4. Rectangular waveguide-to-CPW transition in a back-to-back configuration.

Due to the fabrication process, the cavity has rounded corners with a radius of $200 \mu \mathrm{m}$ and they are included in the design for accuracy improvement. On the quartz substrate two transitions are connected by a CPW in the middle and the air cavity above the CPW has a height of $250 \mu \mathrm{m}$ with the purpose of restricting parasitic modes.

When the proposed wideband patch antenna shown in Fig. 2 is implemented as a rectangular waveguide-to-CPW transition and aluminium is used for the packaging structure, the dimensions of the wideband patch antenna are optimized based on the new environment conditions. Fig. 5 shows the designed wideband patch antenna with optimized dimensions in a back-to-back configuration. The length and width of the quartz substrate are $2238 \mu \mathrm{m}$ and $1050 \mu \mathrm{m}$, respectively. It is also the typical size of the transceiver chips at D-band. Besides, there is no gap between the quartz substrate and the walls of the cavity which helps to restrict parasitic modes and align the wideband patch antenna. The $\mathrm{CPW}$ in the middle of the quartz substrate has a length of $1200 \mu \mathrm{m}$ which includes two antenna feeds and a connection. Unlike the wideband patch antenna designed for wireless communication which has a relatively large ground plane for radiation enhancement, the termination of the rectangular waveguide serves as the bottom ground plane for the optimized wideband patch antenna which results in a more compact design. The total length and width of the patch geometry are reduced to $628 \mu \mathrm{m}$ and $1050 \mu \mathrm{m}$, respectively.

In order to simulate the proposed rectangular waveguideto-CPW transition, wave ports are used as the excitation scheme and they are assigned to the input and output surfaces of the packaging structure. The magnitude of electric field distribution for the packaging structure at $140 \mathrm{GHz}$ is shown in Fig. 6 in which the packaging structure is cut vertically in the middle along the long edge of the quartz substrate. At the beginning of the rectangular waveguide-to-CPW transition, the electric fields going through the tapered waveguide structure are compressed and coupled into the wideband patch antenna. Due to the rectangular ring structure on the patch geometry, the electric fields are concentrated in the slots. Since the wideband patch antennas on the quartz substrate are fed by a CPW, the electric fields mainly exist in the two gaps between the signal and ground traces. With the carefully designed wideband patch antenna as well as the tapered waveguide structure, the proposed rectangular waveguide-to-CPW transition can provide a large bandwidth at D-band which does not introduce

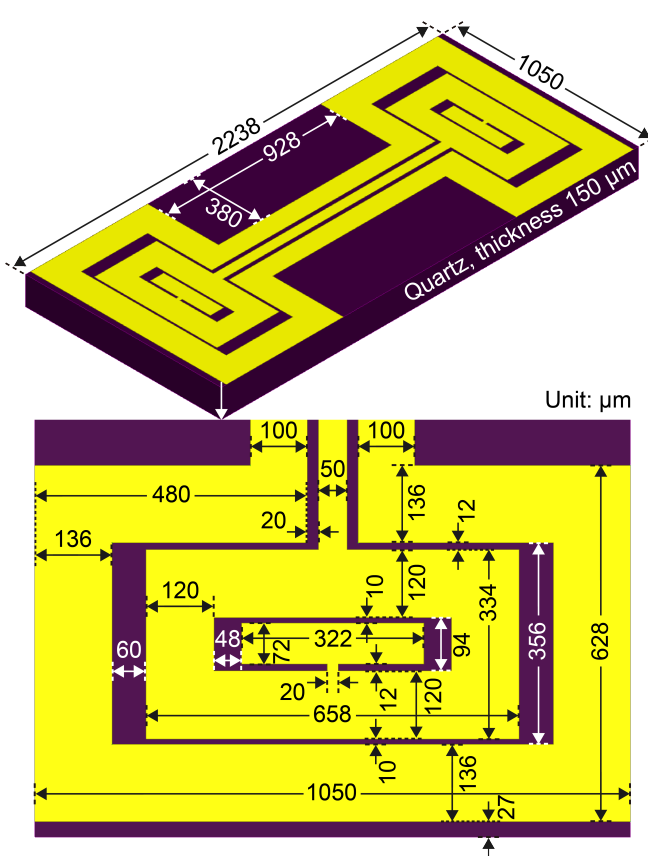

Fig. 5. Designed wideband patch antenna for rectangular waveguide-to-CPW transition.

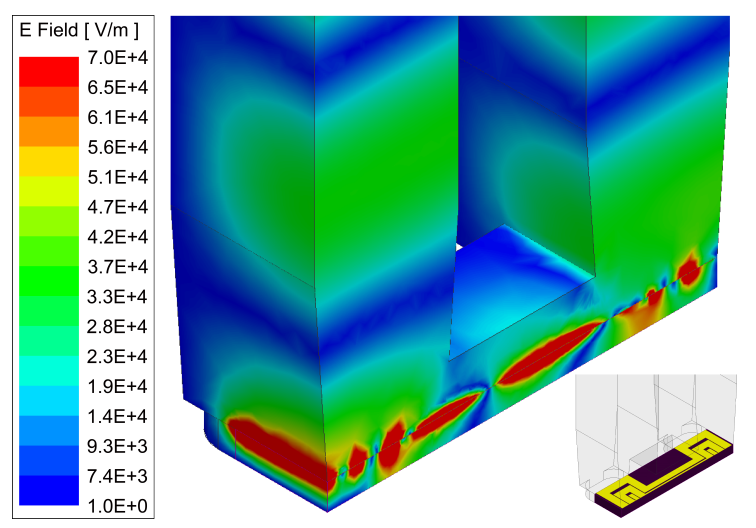

Fig. 6. Magnitude of electric field distribution for the proposed rectangular waveguide-to-CPW transition at $140 \mathrm{GHz}$.

high transmission loss or strong reflection and the propagation mode is converted smoothly.

\section{FABRICATION AND EXPERIMENTAL RESUlTS}

In order to prove the concept, the rectangular waveguide-toCPW transition using wideband patch antenna shown in Fig. 4 was fabricated in a back-to-back configuration. For easier fabrication and substrate alignment, the aluminium packaging structure was divided into three parts and each part was fabricated from an aluminium block by a milling process which uses a spinner with a radius of $200 \mu \mathrm{m}$. Fig. 7 shows the fabricated packaging structure and the quartz substrate in a back-to-back configuration. For assembly, two parts on the top contain the input and output WR-6.5 rectangular waveguides which are connected by using two guide pins and two screws. The cavity for the quartz substrate was milled on the bottom part where four guide pins and four screws are used for combining it with other two parts. 


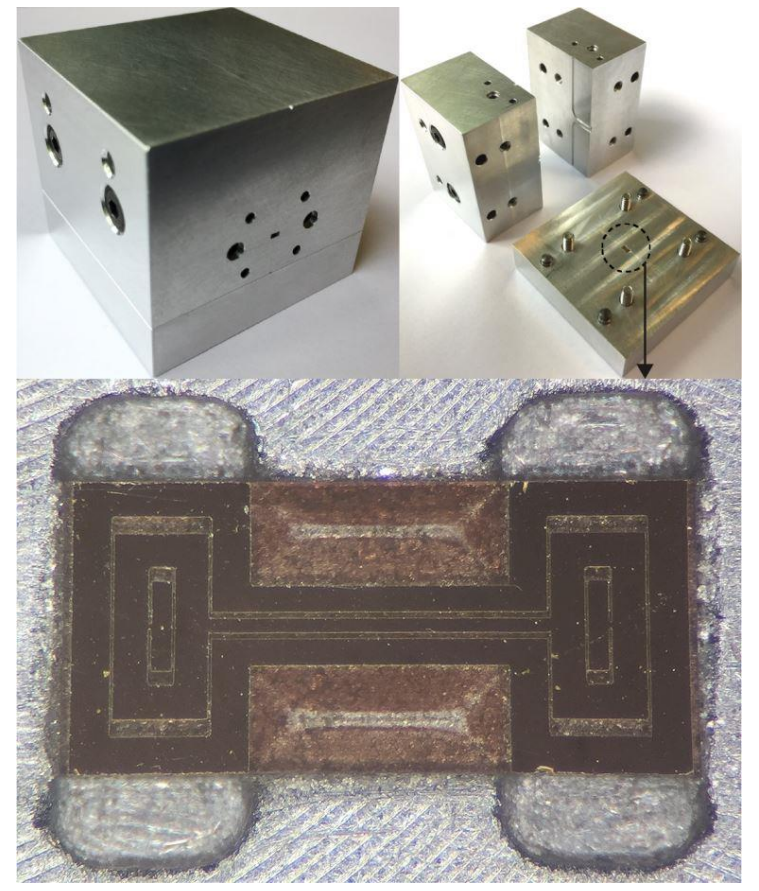

Fig. 7. Fabricated rectangular waveguide-to-CPW transition in a back-to-back configuration.

The proposed transition in a back-to-back configuration was fabricated at Danchip (National Center for Microand Nanofabrication in Denmark). The quartz substrate was cleaned by sputtering at the beginning. After that a titanium layer with a thickness of $30 \mathrm{~nm}$ and a gold layer with a thickness of $400 \mathrm{~nm}$ were deposited on the top where the titanium layer was used to improve the adhesion of the gold layer. The wideband patch antennas and the CPW were patterned on the quartz substrate by laser ablation which uses a picosecond laser with a wavelength of $355 \mathrm{~nm}$ focused down to a spot size of approximate $10 \mu \mathrm{m}$. For dicing process, the patterned quartz substrate was sticked on a silicon wafer in order to overcome the stress. Besides, silver conductive glue was used to fill the gaps between the quartz substrate and the walls of the cavity.

The measurement system consists of an Anritsu ME7808B vector network analyzer (VNA) and two VDI WR-6.5 waveguide extenders which can measure scattering parameters from $110 \mathrm{GHz}$ to $170 \mathrm{GHz}$. For accuracy improvement, throughreflect-line (TRL) calibration was applied by using a waveguide calibration kit and the scattering parameters were calibrated to the input and output of the fabricated packaging structure. The measurement results of the proposed rectangular waveguide-to-CPW transition in a back-to-back configuration are shown in Fig. 8 in comparison with the simulation results. The measured return loss is better than $10 \mathrm{~dB}$ from $118.8 \mathrm{GHz}$ to $161 \mathrm{GHz}$ which corresponds to a bandwidth of $42.2 \mathrm{GHz}$ at D-band. The associated insertion loss is less than $4 \mathrm{~dB}$ for the whole packaging structure which means each fabricated rectangular waveguide-to-CPW transition introduces less than $2 \mathrm{~dB}$ insertion loss. The differences between the measurement and simulation results are mainly caused by the fabrication tolerance of the milling process especially the gaps between different parts of the packaging structure.

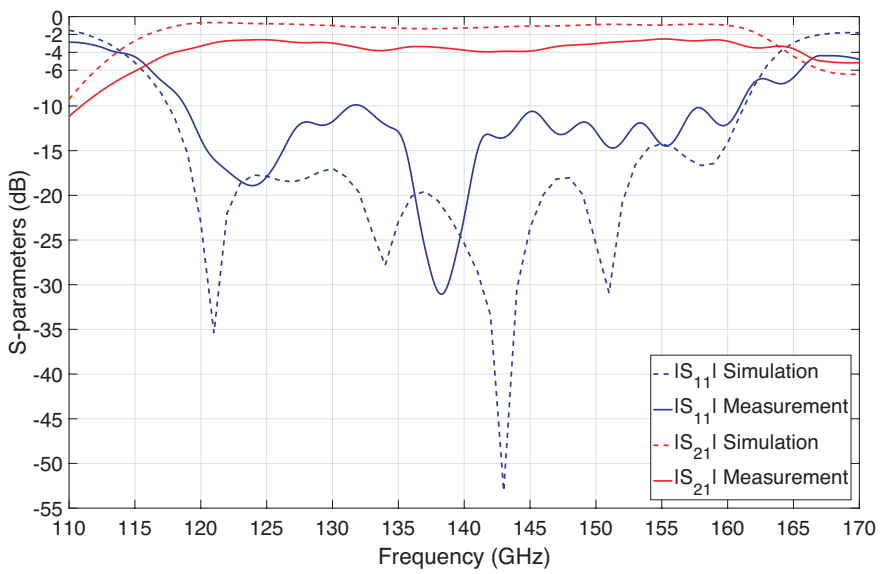

Fig. 8. Simulation and measurement results of the proposed rectangular waveguide-to-CPW transition in a back-to-back configuration.

\section{CONCLUSION}

The designs of wideband patch antennas at D-band used for wireless chip-to-chip communication and rectangular waveguide-to-CPW transition have been presented. For wireless chip-to-chip communication, the proposed wideband patch antenna shows a simulated gain of $7.4 \mathrm{dBi}$ with $36 \%$ bandwidth centered at $140 \mathrm{GHz}$ which corresponds to a bandwidth of $50.3 \mathrm{GHz}$ ranging from $115.3 \mathrm{GHz}$ to $165.6 \mathrm{GHz}$. For system integration and packaging, a rectangular waveguideto-CPW transition in a back-to-back configuration has been designed, fabricated, and measured. The assembly structure has been illustrated in which the aluminium package was divided into three parts. Besides, for accuracy improvement, TRL calibration has been applied to the measurement by using a waveguide calibration kit. The measured return loss is better than $10 \mathrm{~dB}$ from $118.8 \mathrm{GHz}$ to $161 \mathrm{GHz}$ which corresponds to a bandwidth of $42.2 \mathrm{GHz}$ at D-band. Each fabricated rectangular waveguide-to-CPW transition introduces less than $2 \mathrm{~dB}$ insertion loss.

\section{REFERENCES}

[1] J. Xu and D. S. Ricketts. Broadband W-band on-chip Yagi antenna using superstrate for high efficiency and endfire radiation. 2014 Asia-Pacific Microwave Conference, 360-362, Nov 2014.

[2] D. Hou et al. 130-GHz on-chip meander slot antennas with stacked dielectric resonators in standard CMOS technology. Antennas and Propagation, IEEE Transactions on, 60(9):4102-4109, Sept 2012.

[3] M. Neshat, D. M. Hailu, M. R. Nezhad-Ahmadi, G. Z. Rafi, and S. Safavi-Naeini. Gain measurement of embedded on-chip antennas in $\mathrm{mmW} / \mathrm{THz}$ range. Antennas and Propagation, IEEE Transactions on, 60(5):2544-2549, May 2012.

[4] S. Beer, C. Rusch, B. Göttel, H. Gulan, and T. Zwick. D-band gridarray antenna integrated in the lid of a surface-mountable chip-package. 2013 7th European Conference on Antennas and Propagation (EuCAP), 1318-1322, Apr 2013.

[5] Z. J. Hou et al. A $320 \mathrm{GHz}$ on-chip slot antenna array using CBCPW feeding network in $0.13-\mu \mathrm{m}$ SiGe technology. 2017 IEEE MTT-S International Microwave Symposium (IMS), 843-836, June 2017.

[6] V. Zhurbenko et al. Low conversion loss $94 \mathrm{GHz}$ and $188 \mathrm{GHz}$ doublers in InP DHBT technology. 2017 Integrated Nonlinear Microwave and Millimetre-wave Circuits Workshop (INMMiC), 1-3, Apr 2017.

[7] X. Deng, Y. Li, W. Wu, and Y. Xiong. A D-band chip-to-waveguide-horn $(\mathrm{CWH})$ antenna with $18.9 \mathrm{dBi}$ gain using CMOS technology. 2015 IEEE International Wireless Symposium (IWS 2015), 1-4, Mar 2015. 